\title{
Development of rabbit preimplantation blastocysts cultured with precultured endometrial tissue
}

\author{
H-P. Hohn ${ }^{1}$, U. Mootz ${ }^{2}$ and H-W. Denker ${ }^{1}$ \\ ${ }^{1}$ Institut für Anatomie, Universitätsklinikum, Hufelandstr. 55, D-4300 Essen 1, Germany; and \\ ${ }^{2}$ Institut für Anatomie, RWTH, Melatener Straße 211, D-5100 Aachen, Germany
}

\begin{abstract}
Summary. Endometrial fragments were explanted from pseudopregnant rabbits 4.5 days after injecting with human chorionic gonadotrophin and were precultured for 2 days in suspension culture in the presence of oestradiol and progesterone equivalent to concentrations in rabbit serum at that stage. Preimplantation blastocysts were obtained at day 6.5 of pregnancy and cultured in the presence or absence of precultured endometrial fragments. Attachment of the trophoblast to the endometrium was prevented by continuous agitation. After 2 and 3 days, specimens were monitored for development in vitro using light and scanning electron microscopy.

Although the development of blastocysts was slower in vitro than in vivo in both groups, development was clearly superior in the presence of precultured synchronous endometrial fragments. In the absence of endometrium, the embryonic anlage appeared disordered, particularly in the caudal region, but in the presence of uterine tissue the blastocysts developed much better. Up to nine somites were differentiated; the neural tube had started to close and the various parts of the brain anlage showed incipient differentiation. Syncytiotrophoblast differentiated in the presence or absence of endometrium in the embryonic and abembryonic hemispheres, but typical patterns were maintained better and cell degeneration was less frequent during co-culture. Although the culture model described here has not been optimized using criteria of blastocyst differentiation, the results suggest that culture of blastocysts with precultured synchronous endometrial fragments is advantageous.
\end{abstract}

Keywords: blastocyst; endometrium; co-culture; in vitro; rabbit

\section{Introduction}

The survival and development of blastocysts in vivo depend on the availability of an appropriate uterine environment as demonstrated by asynchronous blastocyst transfers (Chang, 1950; Adams, 1965, 1971) and the model of delayed uterine secretion (Beier, 1974). Patterns of uterine protein secretion are subject to stage-specific changes (Beier, 1974, 1982). These changes occur in parallel with specific developmental phases of the blastocyst and are thought to meet specific requirements for the nutrition of the blastocyst. It has been suggested that they are also involved in the regulation of cell differentiation within the blastocyst, but this remains controversial.

The development and differentiation of mammalian blastocysts in vitro has been described in various systems with blastocysts from different species and explantation at different times (Nicholas, 1938; Cole \& Paul, 1965; New, 1967; Gwatkin, 1968; Adams, 1970; Daniel, 1970; New et al., 1973; Hsu, 1978; Edwards et al., 1981; Fisher \& Martinez de Villareal, 1982; Lopata et al., 1983; Sanyal \& Naftolin, 1983; Chávez, 1984; Clarkson et al., 1985; Hansen et al., 1985; Potter \& Morris, 1985; Bavister, 1986; for additional references, see Fischer, 1987). From these investigations, retardation of development in vitro is well known and causes problems, for example in programmes of in vitro 
fertilization, because of the resulting asynchrony between embryo and transformation of the uterus (Beier \& Lindner, 1983). Retardation of development in vitro can be ascribed only partly to the irritation caused by explantation (Seidel, 1960). A possible explanation is that the cited systems generally cannot mimic the nutritional role of the changing uterine environment. Van Blerkom et al. (1973) indicated that at least the first blastocyst stages of rabbit embryos require maternal nutritive factors secreted by the uterus. The advantage of adding uterine flushings to media for blastocyst culture has been demonstrated in various studies in which blastocysts and earlier developmental stages were kept in culture for periods not extending to the implantation phase (in rabbits: El-Banna \& Daniel, 1972a, b; Maurer \& Beier, 1976; McCarthy et al., 1977; Fischer \& Beier, 1986; Fischer, 1987; in mice: Fishel \& Surani, 1978; Sellens \& Sherman, 1980). More recently, Fischer (1987) demonstrated that blastocyst development was influenced more by the stage-specific composition of the uterine secretions added to the culture media than by their quantity. But in all these experiments the uterine flushings were added to the culture medium discontinuously, i.e. the flushing from one phase of pregnancy was provided to developing blastocysts for several days.

Blastocyst cultures on monolayers of uterine and other cells promote embryo development in vitro (Kuzan \& Wright, 1982a, b; Allen \& Wright, 1984; Voelkel et al., 1985; Rexroad \& Powell, 1986; Gandolfi \& Moor, 1987; White et al., 1989; Carney et al., 1990; Menezo et al., 1990) and, although development was still retarded, demonstrated the importance of nutritional factors contributed by the feeder cells for embryo development. Culture with complex fragments of endometrium or of tubal mucosa or in the lumina of uteri or Fallopian tubes after explantation improves blastocyst development (Biggers et al., 1961; Glenister, 1961; Whittingham, 1968; Grant, 1973; Minami et al., 1988). A feature that may not be optimal, however, in all these previously described systems is that detritus of cells damaged during explantation is still present at the time of co-culture and may be noxious. During co-culture within lumina of whole organs in vitro (Grant, 1973), the limited diffusion of nutrients may cause degeneration leading to malfunction of the supporting organ. We expected, therefore, that a recently developed organ culture system would be advantageous in this respect, since it allows removal of most of the cell detritus and restoration of a complete epithelial lining before endometrial fragments are cultured with blastocysts (Hohn $e t$ al., 1989). This endometrial culture system permits certain aspects of progestational transformation to continue in vitro. Assuming that the patterns of endometrial secretion in vitro mimic the situation in vivo, at least to some extent, blastocyst development would be expected to benefit from synchronous culture with such precultured endometrial fragments.

\section{Materials and Methods}

\section{General culture conditions}

Blastocysts and endometrial fragments were cultured in Eagle's minimal essential medium supplemented with penicillin $\left(100 \mathrm{iu} \mathrm{ml^{-1 }}\right)$, streptomycin $\left(100 \mu \mathrm{g} \mathrm{ml}^{-1}\right)$ and non-essential amino acids (MEM-REGA-1: Gibco, Karlsruhe, Germany). The medium was completed immediately before use with $10 \%$ fetal bovine serum (FBS: FBS-309 with certified contents of many hormones, including oestradiol, see below, Gibco, Karlsruhe, Germany), with 3.4 mmol L-glutamine $\mathrm{l}^{-1}$ (Serva, Heidelberg, Germany) and $10 \mathrm{ng}$ progesterone $\mathrm{ml}^{-1}$. Cultures were incubated at $37^{\circ} \mathrm{C}$ on a gyratory shaker in $25 \mathrm{ml}$ Erlenmeyer flasks gassed continuously with $95 \%$ air and $5 \% \mathrm{CO}_{2}$ (modified after Mareel et al., 1979). The media were changed daily.

\section{Endometrial fragments}

Sexually mature rabbits of mixed breeds were kept singly in cages in air-conditioned quarters under a cycle of $12 \mathrm{~h}$ light: $12 \mathrm{~h}$ dark. They were fed a standard pellet food ad libitum. Pseudopregnancy was induced by a single i.v. injection of 75 iu human chorionic gonadotrophin (hCG, Prolan ${ }^{\oplus}$ : Bayer, Leverkusen, Germany). The animals were killed by stunning and exsanguination $4 \cdot 5$ days after hCG injection. Endometrial fragments were obtained from the mesometrial and antimesometrial part of the endometrium and maintained in organ culture at $110 \mathrm{r} . \mathrm{p} . \mathrm{m}$. as previously described by Hohn et al. (1989). Oestrogen concentrations in the media within the range in rabbit serum were provided by the FBS 
additive; progesterone $\left(10 \mathrm{ng} \mathrm{ml}^{-1}\right)$ was added to the culture medium to induce gestational transformation as judged by morphological criteria. After 2 days $(6.5$ days after $\mathrm{hCG})$, such precultured endometrial fragments were put in co-culture with blastocysts of the same stage (Fig. 1).

\section{Blastocysts and co-culture}

On day 0 of an experiment (Fig. 1), oestrous females were mated with two males and ovulation was ensured by an intravenous injection of 75 iu hCG. At 6.5 days post coitum ( $156 \mathrm{~h}$ post coitum) the animals were killed by stunning and exsanguination. The uteri were explanted immediately and the embryo-containing uterine swellings were cut out with scissors. The blastocysts were obtained by carefully tearing apart the antimesometrial part of the uterine wall with fine surgical forceps and were transferred into culture medium with intact blastocyst coverings. They were cultured in Erlenmeyer flasks as described, with the shaker set at 60 r.p.m., in the presence or absence of precultured endometrial fragments ( 30 fragments per flask). In contrast to previous experiments (Hohn \& Denker, 1990), close contact between blastocysts and endometrial fragments was prevented by continuous shaking throughout the culture period, so that both parts were kept free-floating to avoid attachment. Results were evaluated morphologically after 2 and 3 days in culture, days 8.5 and 9.5 post coitum, respectively. As a control, the uteri of pregnant animals were excised at corresponding times post coitum and, after opening up the implantation chamber, the embryonic anlage was dissected with fine scissors and processed for examination.

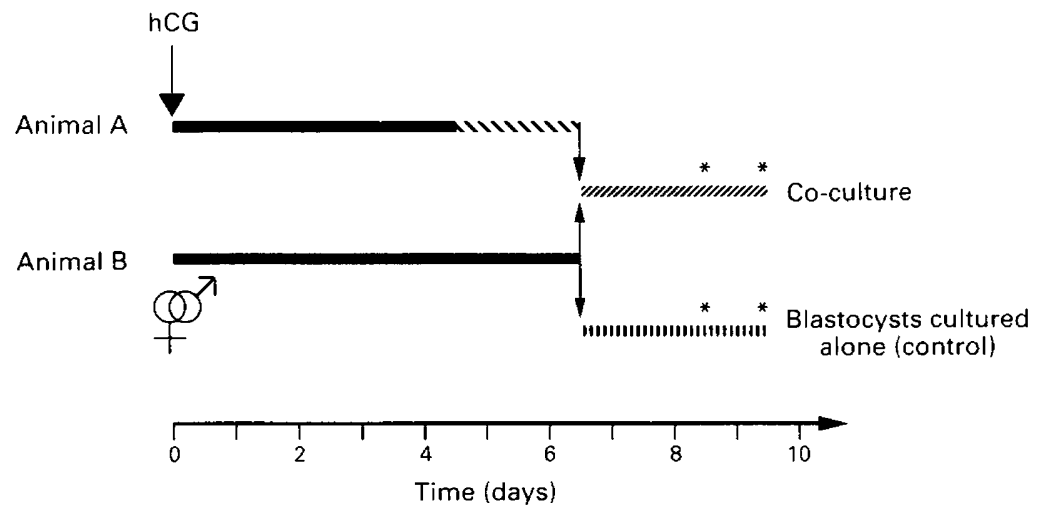

Fig. 1. Experimental design. Animal (rabbit) A: donor of endometrial fragments. Pseudopregnancy was induced by i.v. injection with human chorionic gonadotrophin (hCG). Animal B: donor of blastocysts. $\mathbf{D}$ in vivo; endometrial organ culture; 四 blastocyst culture; co-culture of blastocysts and endometrial fragments; *examination of blastocyst development.

\section{Morphology}

After culture the blastocysts were transferred into $2.5 \%$ glutaraldehyde in $0 \cdot 1$ mol cacodylate buffer $1^{-1}(\mathrm{pH} 7 \cdot 4)$ for at least $4 \mathrm{~h}$. During this time developmental patterns were evaluated and photographs were taken using a Wild Photomakroskop M 400. The blastocyst coverings were removed with fine surgical forceps and microsurgical scissors to facilitate optimal fixation. The embryonic and abembryonic hemispheres were separated at the equatorial line. After fixation the blastocysts were rinsed in $0.1 \mathrm{~mol}$ cacodylate buffer $1^{-1}$ overnight. The specimens were stained with toluidine blue $\left(0.1 \%, w / v\right.$, in $0.1 \mathrm{~mol}$ cacodylate buffer $1^{-1}, \mathrm{pH} \mathrm{7.4)}$ for easier handling under the dissecting microscope. To prepare them for scanning electron microscopy, subsequently, radial cuts were made from the periphery of the embryonic anlage to the edge of the embryonic half to permit flat mounting. In the abembryonic part, the cuts extended across one-third of the diameter. The specimens were then flattened on small cork plates, mounted with fine needles and gradually dehydrated in acetone ( $10 \%$ to $50 \%$ for $30 \mathrm{~min}$ each; $60 \%$ to $100 \%$ for $2 \mathrm{~h}$ each). After dehydration the material was dried to the critical point in liquid $\mathrm{CO}_{2}$ and sputter-coated with gold. Scanning electron microscopy was performed in a Leitz AMR 1000A.

After fixation some of the blastocysts were fixed in $2 \%(\mathrm{w} / \mathrm{v})$ osmium tetroxide, dehydrated in a graded series of ethanol and embedded in araldite. Semi-thin sections $(0.75 \mu \mathrm{m})$ were stained with $0.5 \%$ toluidine blue and $0.5 \%(\mathrm{w} / \mathrm{v})$ pyronin in $0.5 \%(\mathrm{w} / \mathrm{v})$ sodium borate. Sections were examined and photographed with a Zeiss Photomicroscope II. 


\section{Results}

\section{State of control embryos}

Blastocysts before culture (day 6.5 post coitum) and control embryos explanted at days 8.5 and 9.5 post coitum showed some variation in size and developmental state within the ranges defined by Seidel (1960) and by Gottschewski \& Zimmermann (1973).

\section{General effects during blastocyst culture}

Rabbit blastocysts expand extensively during the peri-implantation phase and are therefore delicate to handle. This feature resulted in the loss of some blastocysts during explantation, when the diameter is up to $5 \mathrm{~mm}$. During subsequent culture, the diameter increased to $15 \mathrm{~mm}$ after 3 days in culture (corresponding to day 9.5 in vivo). As a result of this expansion the blastocysts became increasingly fragile and some collapsed partly or completely in culture. Of 56 blastocysts observed during these experiments, only $31 \%$ were not collapsed after 2 or 3 days in culture, and were taken for further examination of developmental stage (see Table 1).

Table 1. Development of rabbit blastocysts in vitro in the presence $(+)$ or absence $(-)$ of precultured endometrial fragments

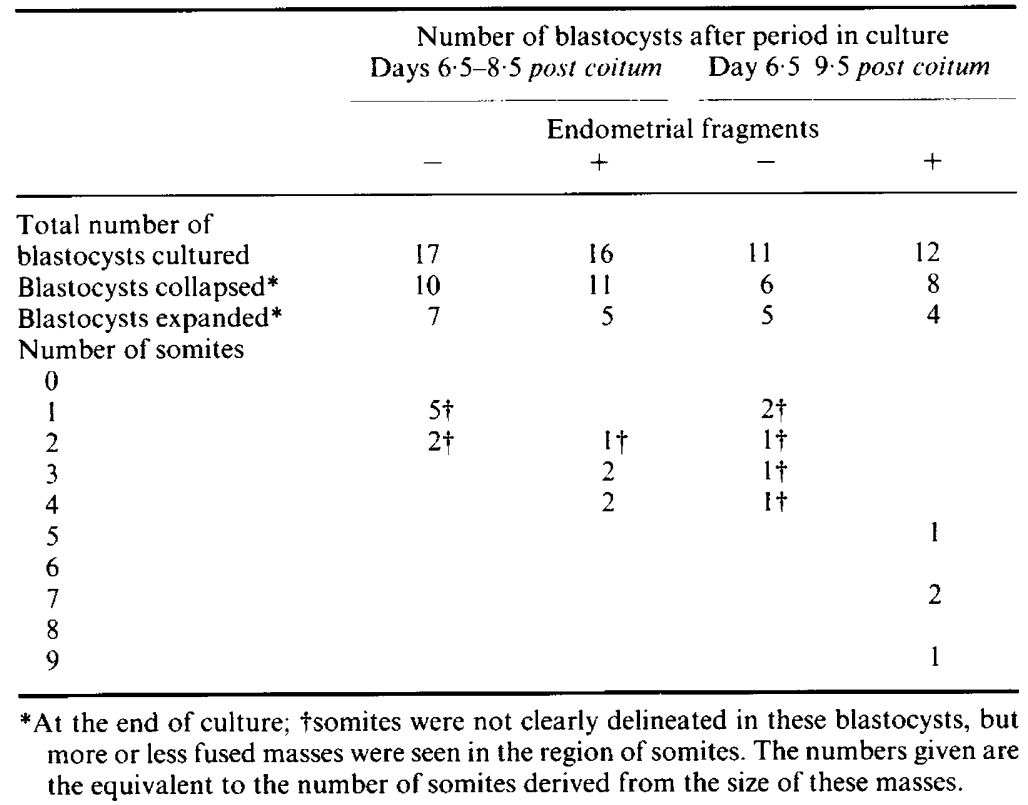

\section{Embryonic anlage}

Development of the embryo in vitro was retarded in these experiments as reported for all other culture models, but there were considerable differences between both series of experiments.

In the absence of endometrial fragments. The development of the blastocysts was not only delayed but also severely disorganized resulting in serious degeneration (Fig. 2) in almost all parts of the embryonic anlage, morphology usually being distorted most severely in the caudal region (Figs 2 and 3). The embryonic anlage of these blastocysts was significantly smaller than that of 
control embryos developed for the same time in vivo. Often the identification of anatomical details was difficult by light or scanning electron microscopy. It was hardly possible to distinguish individual somites in blastocysts grown in the absence of endometrium (Fig. 3a, Table 1). Neural folds and the neural crest developed in some specimens, but closure of the neural tube was not observed. Amniotic folds appeared only rudimentarily.
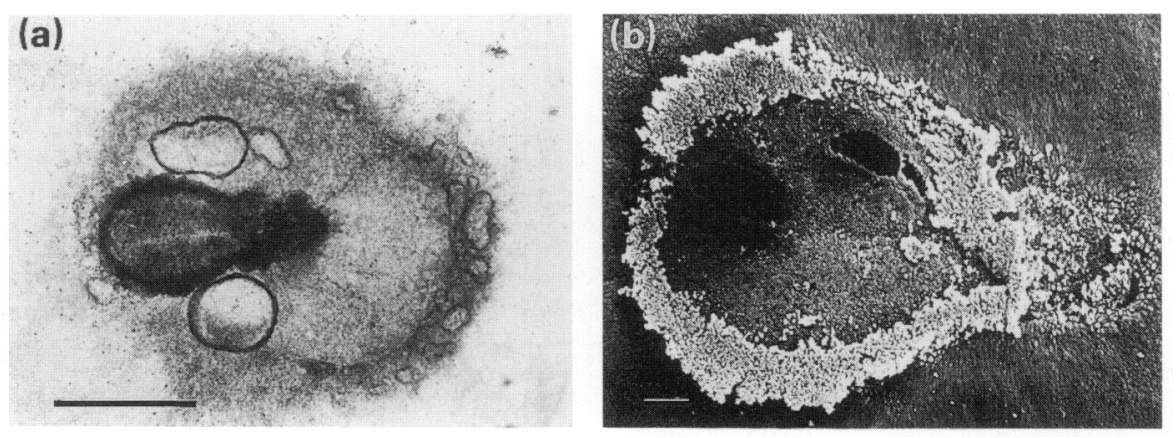

Fig. 2. After maintenance (days $6 \cdot 5-8 \cdot 5$ post coitum) of rabbit blastocysts in culture medium alone (control) this embryonic anlage shows (a) degeneration (appearing as inflated, vesicular structures) in the mesenchyme (light microscopy) and (b) serious cell shedding in all regions, which is more pronounced in the caudal area (scanning electron microscopy). Bars represent (a) $1 \mathrm{~mm}$, (b) $0.1 \mathrm{~mm}$.
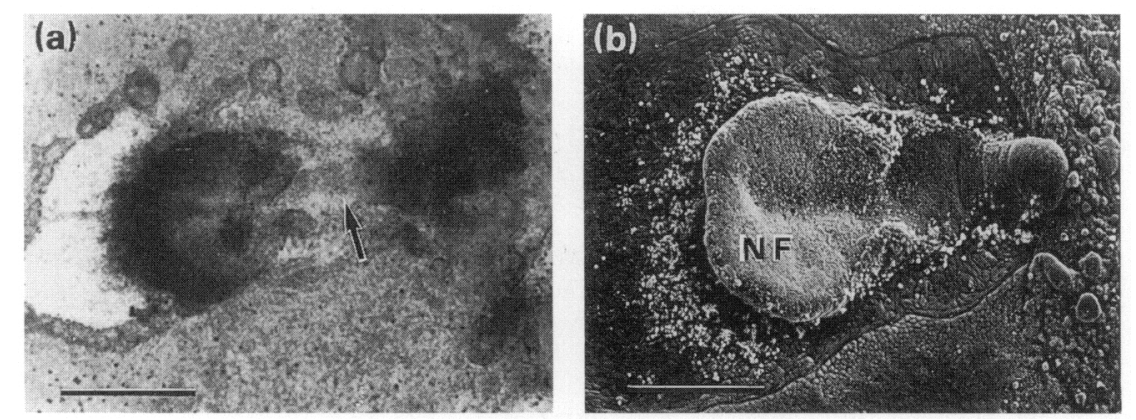

Fig. 3. After 3 days (equivalent to day $9 \cdot 5$ post coitum) in medium alone (control) (a) light microscopy of this rabbit embryo reveals only minor traces of what may be somites (arrow). (b) Scanning electron microscopy demonstrates asynchronous development with respect to the cranial and the caudal (right) regions. The neural folds (NF) have only reached a state of differentiation according to 8 days post coitum in vivo. Failure of amniotic fold development resulted in exposure of the rudimentarily developed caudal area. Bars represent $0.5 \mathrm{~mm}$.

Blastocysts in co-culture with precultured endometrial fragments. These blastocysts developed much better than those in culture medium alone. In some respects development was only slightly slower than in the control embryos and for the norm table for rabbit development presented by Seidel (1960), although major distortions were observed in some specimens. After 2 days in culture (day 8.5 post coitum), they showed up to four somites (Fig. $4 a, b$ ), the neural tube appeared, but was still wide open, and the brain was folding up (Fig. 4b). The proamniotic area could also be identified. Cross-sections demonstrated that the organization of the germ layers was well preserved in this system (Fig. 5). After 3 days in vitro (day 9.5 post coitum) development had proceeded further, but as referred to the table of Seidel (1960) and to the control embryos, relative retardation 
of development was more obvious than on day 2 in culture. The developmental state that was reached corresponded roughly to embryos at days $8 \cdot 5-9$ post coitum: up to nine somites (Table 1), neural tube near to closing, and amniotic folds enlarged and moving towards each other. One specimen showed, in the lateral cranial areas, what appeared to be the otic placode, and the heart anlage was protruding (Fig. 6).
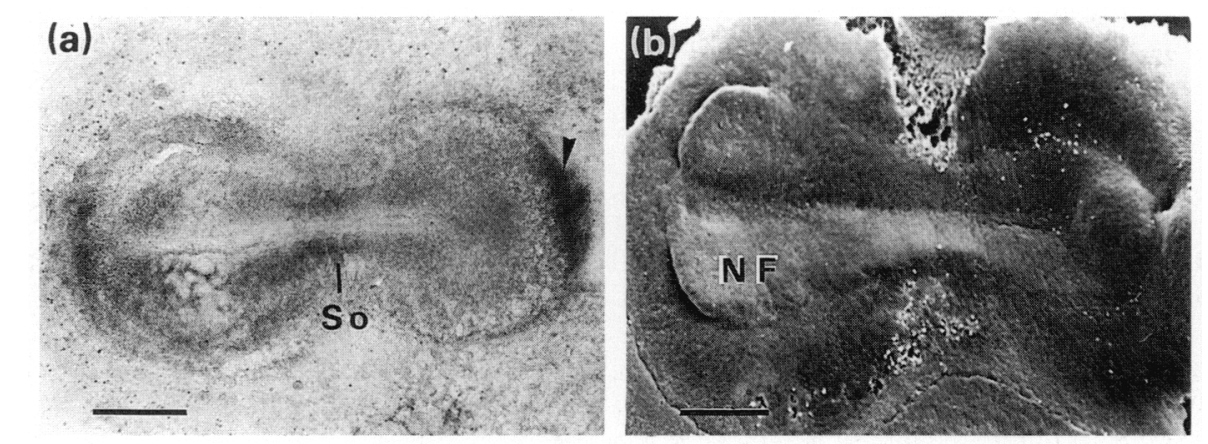

Fig. 4. A rabbit embryo co-cultured with endometrial fragments for 2 days (days 6.5-8.5 post coitum) shows harmonic overall development as shown by (a) light microscopy and (b) scanning electron microscopy. There are somites (So) and well-differentiated neural folds (NF). The posterior amniotic fold is also discernible (arrowhead). Bars represent $0.5 \mathrm{~mm}$.

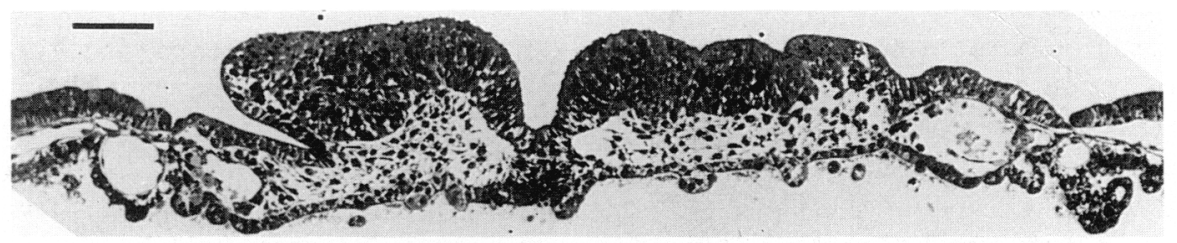

Fig. 5. Rabbit embryonic anlage cultured with endometrium for 2 days. Cross-section in the posterior area of the neural plate/fold with neural groove and primitive streak. The mesenchyme showing several blood vessels is well preserved. Bar represents $0 \cdot 1 \mathrm{~mm}$.

Sloughing of degenerated cells and cellular debris was seen more often in the absence of endometrial fragments than during co-culture. This phenomenon occurred predominantly in the trophoblast (particularly in the abembryonic syncytiotrophoblast).

\section{Trophoblast}

Attachment of the blastocyst (via the trophoblast) to co-cultured endometrium was avoided by continuous agitation and was not observed in these experiments. Differentiation of the trophoblast did proceed, in morphological terms, both in pure blastocyst culture and in co-culture with endometrium.

In the abembryonic trophoblast, numerous trophoblastic knobs containing small syncytial elements developed in culture (Figs 7 and 8). The subdivision into cytotrophoblast and syncytiotrophoblast was clearly maintained until the end of the observation period, i.e. day $9 \cdot 5$ post coitum. Because in these cultures the trophoblast did not attach to the endometrium (as it does in vivo) it was possible to study the dimensions of trophoblastic knobs in flat mounts up to late stages that cannot be studied in the same way after implantation in vivo. In vitro the trophoblastic knobs tended to aggregate and to fuse with each other progressively towards the end of culture. Aggregation of 

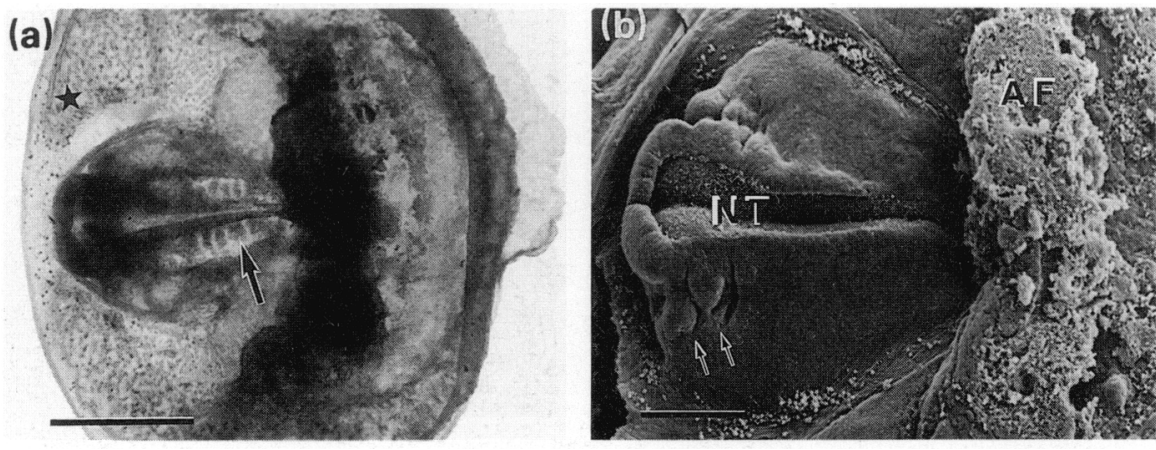

Fig. 6. This embryonic anlage of a rabbit blastocyst that has been in endometrial co-culture for 3 days (until day $9 \cdot 5$ post coitum) demonstrates that development has progressed well and is only slightly retarded. (a) Several pairs of somites (arrow, light microscopy) are visible; $\star$ anterior limits of the horseshoe-shaped syncytial mass of the trophoblast at the embryonic pole. (b) The neural tube (NT) is in the process of closing, and the amniotic folds are moving towards each other; AF, caudal amniotic fold (scanning electron microscopy). In the cranial lateral regions the surface exhibits symmetrical structures (double arrows) that may represent branchial arches. Bars represent: (a) $1 \mathrm{~mm}$, (b) $0.5 \mathrm{~mm}$.

trophoblastic knobs was first seen in a ring-shaped region surrounding the abembryonic pole and continued towards that pole until the polar area originally free of trophoblast knobs had disappeared. This condensation was more or less complete after 3 days in culture in the absence of endometrium, but in the presence of endometrium the separation of cytotrophoblast and syncytiotrophoblast elements was preserved better (Fig. 7). Such differences between the two types of culture were not seen within the first 2 days of culture.

At the embryonic pole, syncytiotrophoblast differentiated in much the same way as in vivo, in the presence of endometrium. It formed a large horseshoe-shaped plaque incompletely surrounding the embryonic anlage (Fig. 9). A thick layer of syncytiotrophoblast developed on top of a thin layer of cytotrophoblast cells (Fig. 10). When blastocysts were cultured alone differentiation of the embryonic syncytiotrophoblast proceeded more slowly covering a smaller area after the same time in culture.

\section{Discussion}

Previous attempts at supplying components of uterine secretion to blastocysts during culture in vitro have used a discontinuous system where uterine flushings from one phase of pregnancy were provided to developing blastocysts in vitro for several days so that the continuously changing patterns of secretion in the uterus are not mimicked. The experiments reported here try to take these drawbacks into consideration by maintaining preimplantation rabbit blastocysts in synchronous co-culture with precultured rabbit endometrial fragments taking advantage of a previously developed novel system for endometrial organ culture (Hohn et al., 1989). The data presented demonstrated that embryo development is supported better in this system than in culture medium alone, as seen most clearly with respect to the differentiation of the embryo as well as of the invasive elements of the trophoblast. Beneficial effects may result from (i) factors secreted by the endometrium, (ii) from removal of detrimental metabolites of the blastocyst by the endometrium or (iii) from direct cell interactions during occasional contacts of the blastocyst with the endometrium. The co-culture of blastocysts with cell monolayers has been shown to support blastocyst development by Cole \& Paul (1965), Allen \& Wright (1984) and Chávez (1984) who used endometrial cells as well as other feeder cells. The development obtained in the co-culture system described here was not compared in 

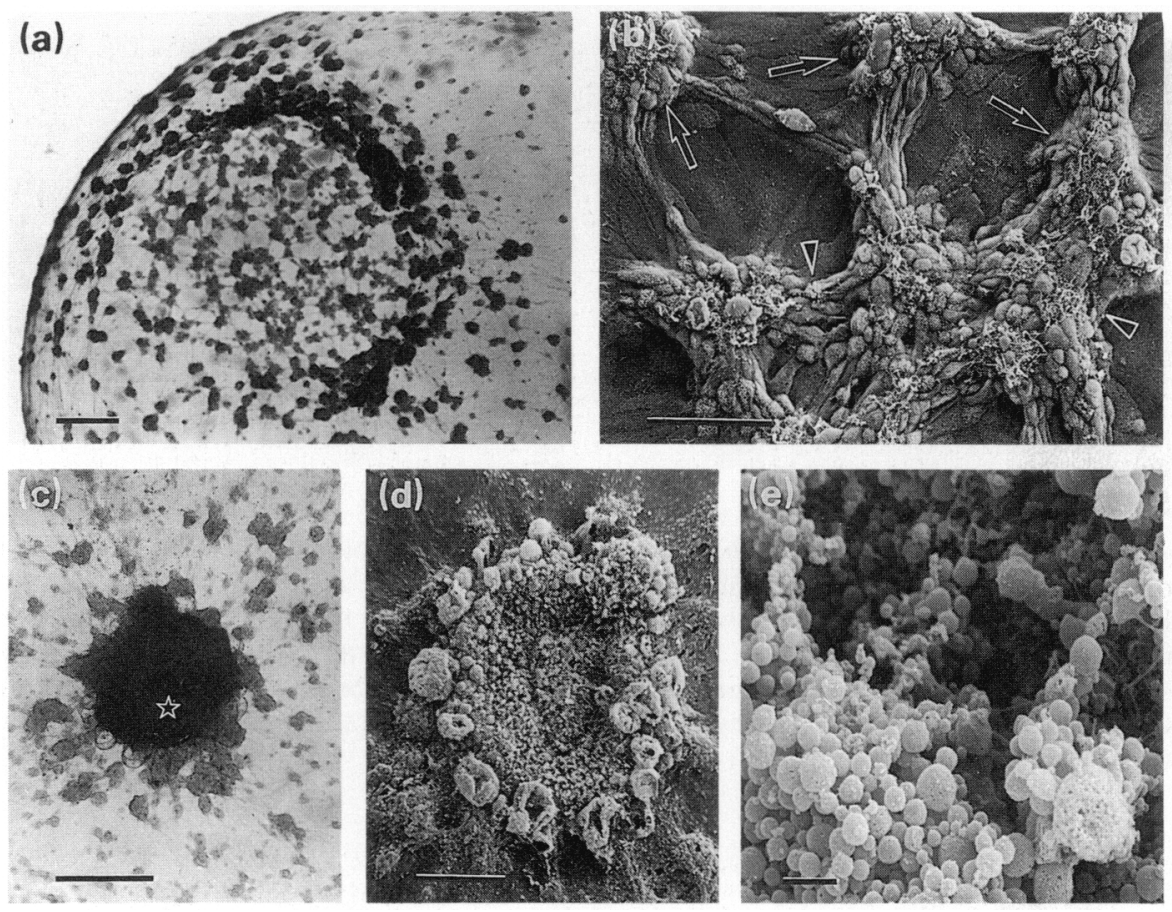

Fig. 7. State of development of the abembryonic trophoblast of rabbit blastocysts after maintenance in vitro for 3 days (days 6.5-9.5 post coitum). Numerous trophoblastic knobs are observed $(a, b)$ in the presence and $(c, d, e)$ the absence of endometrial fragments. Only limited signs of degeneration were observed in culture, particularly in the trophoblastic knobs $(b$, arrows). In the absence of trophoblast attachment, which was prevented, condensation is seen to occur resulting in confluence of trophoblastic knobs (arrowheads). In co-culture $(a, b)$, demarcation of individual trophoblastic knobs with interposed cytotrophoblast is clearly preserved. In the absence of endometrium (c, d, e), trophoblastic knobs tend to show more extensive aggregation at the abembryonic pole (c d). This mass of aggregated tissuc shows a high degree of cell degeneration (e, higher magnification from the centre of $d$ ) and cytotrophoblast and syncytiotrophoblast can no longer be distinguished. $(a, c)$ light microscopy, $(b, d, c)$ scanning electron microscopy. Bars represent (a) $1 \mathrm{~mm}$, (b) $0.1 \mathrm{~mm}$, (c) $1 \mathrm{~mm}$, (d) $0.5 \mathrm{~mm}$, (c) $20 \mu \mathrm{m}$.

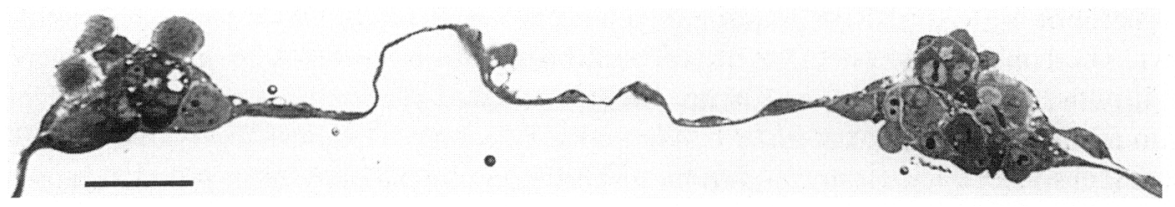

Fig. 8. Cross-section of rabbit abembryonic trophoblast after culture with endometrial fragments for 2 days. Two trophoblastic knobs consisting of cytotrophoblast and small syncytiot rophoblast elements are well separated by an area of thin cytotrophoblast cells. Bar represents $50 \mu \mathrm{m}$.

parallel experiments with the effects of co-culturing blastocysts and cell monolayers. However, comparison with reports by other authors (Kuzan \& Wright, 1982a, b; Allen \& Wright, 1984; Voelkel et al., 1985; Rexroad \& Powell, 1986; Gandolfi \& Moor, 1987; White et al., 1989; Carney et al., 1990; Menezo et al., 1990) on co-culture with monolayers in other species suggests that blastocysts developed remarkably well in our system, perhaps because differentiation and other 

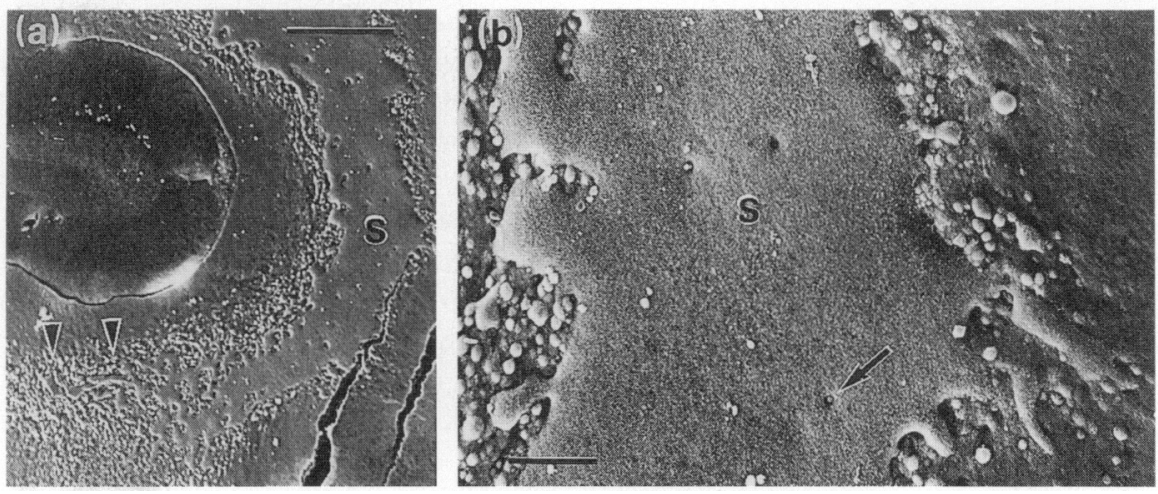

Fig. 9. Differentiation of rabbit embryonic syncytiotrophoblast after 2 days in culture with endometrium. As implantation does not occur in this culture system, the cell fusion in the area of the embryonic syncytiotrophoblast proceeds until the caudal region of the embryo is surrounded by a gigantic horseshoe-shaped syncytial mass ( $\mathrm{S}$ in (a) and (b); arrowheads indicate the anterior end, (b) is a higher magnification of (a) in the area of S; see also Fig. 6a). Individual cells are found mainly at the border but rarely interposed within this mass (b, arrows). Bars represent: (a) $0.5 \mathrm{~mm}$, (b) $0.1 \mathrm{~mm}$.

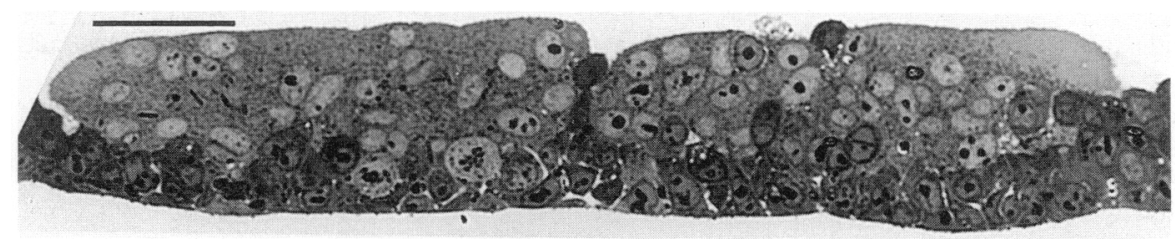

Fig. 10. After 2 days in endometrial co-culture, the rabbit trophoblast at the embryonic pole is well differentiated into a thick layer of syncytiotrophoblast covering a basal layer of cytotrophoblast cells. Single individual cells are still found within and on top of the syncytiotrophoblast. Mitoses are seen in the cytotrophoblast. The bar represents $50 \mu \mathrm{m}$.

functional characteristics of the endometrium were particularly well expressed in our organ cultures (Hohn et al., 1989; Hohn \& Denker, 1990). A specific feature of this organ culture model is that it attempts to maintain in vitro the epithelio-stromal interactions known to be important for the action of steroid hormones (Cunha, 1985; Bigsby \& Cunha, 1986). However, the pattern of protein secretion by endometrial fragments in culture has not been investigated and, therefore, it is unknown whether blastocyst development reflects the differential expression of specific proteins or general beneficial properties of this 'feeder tissue'.

Although blastocysts differentiated well in our experiments in co-culture with endometrium, they were still somewhat retarded and did not reach quite the same stages within the same period as in vivo. In addition, there was some cell degeneration and sloughing, probably because (i) of adverse effects of explantation on blastocysts (i.e. before putting them in culture) and (ii) our culture conditions may still be suboptimal. Procedures used for explanting blastocysts and handling them in vitro obviously cause some retardation and damage, as shown by previous investigations. Studies of embryo transfers (Seidel, 1960) suggest that retardation of about 0.5 days is caused by explanting blastocysts. During explantation, a blastocyst is exposed to mechanical irritation and oscillations in temperature and light. Daniel (1964) and Schumacher \& Fischer (1988) showed that the exposure to light induces retardation in embryo development. However, as the pattern of protein secretion has not been monitored in the endometrial culture system used here, retardation in embryo development may be caused by delayed or insufficient secretion of important factors. 
Further improvement of blastocyst development may be achieved by optimizing, in this system, the culture conditions in terms of media, culture device, aeration and pretreatment of blastocysts before culture. The culture medium used in the present experiments was the same as that chosen previously for the endometrial organ culture system (Hohn et al., 1989). Although this kind of media containing vitamins and amino acids is similar to those recommended for blastocyst culture by Daniel \& Krishnan (1967), Kane \& Foote (1970a, b, 1971) and Kane \& Headon (1980), it may not be optimal for co-culture with endometrium. The advantage of supplementing the medium with oestrogens and progesterone at concentrations similar to those used here was demonstrated by Fisher \& Martinez de Villareal (1982) and by El-Banna \& Daniel (1972a). The medium might be improved by a better selection of the serum supplement, as indicated by Lear et al. (1983). The ratio of endometrial fragments to blastocysts in a certain volume of medium could be important for achieving an optimal concentration of secreted factors.

A further improvement of the medium might be the addition of other growth factors or vitamins, maintenance of a $\mathrm{pH}$ according to the uterine environment or of an appropriate $\mathrm{CO}_{2}: \mathrm{O}_{2}$ ratio. An apparatus for the culture of blastocysts in a continuous flow of medium alone was introduced by Nicholas (1938) and modified by New \& Daniel (1969) using rat embryos. It was adapted for the culture of rabbit blastocysts by Daniel (1970), with a high degree of development when blastocysts were cultured from day 6 and $16 \mathrm{~h}$ to day 9 and $16 \mathrm{~h}$ post coitum. Blastocyst development obtained in these experiments was comparable to our co-culture experiments; but this device may hardly be used in a co-culture model since the endometrial secretion would be diluted continuously. Good results were also obtained in roller bottle systems (Sanyal \& Naftolin, 1983; New et al., 1973), indicating the advantage of agitating the free floating blastocysts, which was also reported by Cole \& Paul (1965). Pretreatment of blastocysts before culture to alleviate their hatching is advised by El-Banna \& Daniel (1972a), who obtained better growth of blastocysts after incubation of the blastocyst coverings with pronase. Daniel (1970) also observed better development after spontaneous hatching of blastocysts than when blastocysts remained within their coverings.

These results indicate that the co-culture system described here using precultured endometrial fragments may be of advantage for a number of applications in which continuing differentiation of blastocysts in vitro is required, e.g. in basic research on the differentiation of germ layers in mammalian embryos and for certain regimens of in vitro fertilization and programmes of embryo transfer. Co-culture with endometrium as presented here appears to be of value particularly for the culture of blastocysts (including advanced stages). It should also be easy to modify and optimize this system for the culture of early cleavage stages by using similarly precultured tubal mucosa instead.

The authors wish to thank G. Helm and E. Hölscher for excellent technical assistance, and G. Bock (Institute of Anatomy, Aachen) and the photography staff of the Institute of Anatomy in Essen for photographic work. Special thanks are due to H. van Straaten in Maastricht (Netherlands) for discussing the results. The investigations were supported by DFG grants De 181/9-6 and Ho $1059 / 1-7$.

\section{References}

Adams, C.E. (1965) The influence of maternal environment on preimplantation stages of pregnancy in vitro. In Preimplantation Stages of Pregnancy, pp. 345-373. Eds G. E. W. Wolstenholme \& M. O'Connor. Churchill, London.

Adams, C.E. (1970) The development of rabbit eggs after culture in vitro for 1-4 days. Journal of Embryology and Experimental Morphology 23, 21-34.

Adams, C.E. (1971) The fate of fertilized eggs transferred to the uterus or oviduct during advancing pseudo- pregnancy in the rabbit. Journal of Reproduction and Fertility 26, 99-111.

Allen, R.L. \& Wright, R.W., Jr (1984) In vitro development of porcine embryos in coculture with endometrial monolayers and culture supernatants. Journal of Animal Science 59, 1657-1661.

Bavister, B.D. (1986) In Developmental Biology: a Comprehensive Synthesis, Vol. 4: Manipulation of Mammalian Development, pp. 81-147. Ed. R. B. L. Gwatkin. Plenum Press, New York and London. 
Beier, H.M. (1974) Oviducal and uterine fiuids. Journal of Reproduction and Fertility 37, 221-237.

Beier, H.M. (1982) Uteroglobin and other endometrial proteins: biochemistry and biological significance in beginning pregnancy. In Proteins and Steroids in Early Pregnancy, pp. 39-71. Eds H. M. Beier \& P. Karlson. Springer-Verlag, Berlin.

Beier, H.M. \& Lindner, H.R. (1983) Fertilization of the Human Egg In Vitro. Springer Verlag, Berlin.

Bigsby, R.M. \& Cunha, G.R. (1986) Estrogen stimulation of deoxyribonucleic acid synthesis in uterine epithelial cells which lack estrogen receptors. Endocrinology 119, 390-396.

Biggers, J.D., Gwatkin, R.B.L. \& Brinster, R.L. (1961) Development of mouse embryos in organ culture of Fallopian tubes on a chemically defined medium. Nature 194, 747-749.

Carney, E.W., Tobback, C. \& Foote, R.H. (1990) Coculture of rabbit one-cell embryos with rabbit oviduct epithelial cells. In Vitro Cellular and Developmental Biology 26, 629-635.

Chang, M.C. (1950) Development and fate of transferred rabbit ova or blastocyst in relation to the ovulation time of recipients. Journal of Experimental Zoology 114, 197-225.

Chávez, D.J. (1984) Embryology of the mouse from ovulation through periimplantation stages in vitro. Scanning Electron Microscope 11, 729-735.

Clarkson, J.L., Richardson, M.E., Dickey, J.F. \& Halman, R.D. (1985) Effects of medium removed from endometrial cell cultures on in vitro growth of ovine embryos. Journal of Animal Science Supplement I, 409 (Abstract).

Cole, R.J. \& Paul, J. (1965) Properties of preimplantation mouse and rabbit embryos, and cell strains derived from them. In Preimplantation Stages in Pregnancy, pp. 82-122. Eds G. E. W. Wolstenholme \& $\mathbf{M}$. O'Connor. Little, Brown \& Co., Boston.

Cunha, G.R. (1985) Mesenchymal-epithelial interactions during androgen-induced development of the prostate. In Developmental Mechanisms: Normal and Abnormal, pp. 15-24. Eds J. W. Lash \& L. Saxén. Alan R. Liss Inc, New York.

Daniel, J.C., Jr (1964) Cleavage of mammalian ova inhibited by visible light. Nature 201, 316-317.

Daniel, J.C., Jr (1970) Culture of rabbit embryos in circulating medium. Nature 225, 193-194.

Daniel, J.C. \& Krishnan, R.S. (1967) Amino acid requirements for growth of the rabbit blastocyst in vitro. Journal of Cell Physiology 70, 155-160.

Edwards, R.G., Purdy, J.M., Steptoe, P.C. \& Walters, D.E. (1981) The growth of human preimplantation embryos in vitro. American Journal of Obstetrics and Ginecology 141, 408-416.

El-Banna, A.A. \& Daniel, J.C., Jr (1972a) Stimulation of rabbit blastocysts in vitro by progesterone and uterine proteins in combination. Fertility and Sterility 23, 101-104.

El-Banna, A.A. \& Daniel, J.C., Jr (1972b) The effect of protein fractions from rabbit uterine fluids on embryo growth and uptake of nucleic acid and protein precursors. Fertility and Sterility 23, 105-114.

Fischer, B. (1987) Development retardation in cultured preimplantation rabbit embryos. Journal of Reproduction and Fertility 79, 115-123.
Fischer, B. \& Beier, H.M. (1986) In-vitro-Kultur von Präimplantations-embryonen. Entwicklungskriterien und Uterussekretzusatz zum Kulturmedium. Fertilität 2, 97-103.

Fishel, S.B. \& Surani, A.H. (1978) Changes in responsiveness of preimplantation mouse embryos to serum. Journal of Embryology and Experimental Morphology 45, 295-301.

Fisher, D.L. \& Martinez de Villareal, L. (1982) Effects of hormones on postimplantation mouse embryos in vitro. II. Progesterone and estrogen. Journal of Experimental Zoology 224, 205-210.

Gandolfi, F. \& Moor, R.M. (1987) Stimulation of early embryonic development in the sheep by co-culture with oviductal epithelial cells. Journal of Reproduction and Fertility 81, 23-28.

Glenister, T.W. (1961) Observations on the behaviour in organ culture of rabbit trophoblast from implanting blastocysts and early placentae. Journal of Anatomy 95, $474-485$.

Gottschewski, G.H.M. \& Zimmermann, W. (1973) Die Embryonalentwicklung des HauskaninchensNormogenese und Teratogenese. Verlag M. \& $\mathrm{H}$. Schaper, Hannover.

Grant, P.S. (1973) The effect of progesterone and oestradiol on blastocysts cultured within the lumina of immature mouse uteri. Journal of Embryology and Experimental Zoology 29, 617-638.

Gwatkin, R.B.L. (1968) Amino acid requirements for attachment and outgrowth of the mouse blastocyst in vitro. Journal of Cell Physiology 68, 335-343.

Hansen, P.J., Anthony, R.V., Bazer, F.W., Baumbach, G.A. \& Roberts, R.M. (1985) In vitro synthesis and secretion of ovine trophoblast protein-1 during the period of maternal recognition of pregnancy. Endocrinology 117, 1424-1430.

Hohn, H-P. \& Denker, H-W. (1990) A three-dimensional organ culture model for the study of implantation of rabbit blastocysts in vitro. Trophoblast Research 4, 75-95.

Hohn, H-P., Winterhager, E., Busch, L.C., Mareel, M.M. \& Denker, H-W. (1989) Rabbit endometrium in organ culture: morphological evidence for progestational differentiation in vitro. Cell and Tissue Research 257, 505-518.

Hsu, Y.C. (1978) In vitro development of whole mouse embryos beyond the implantation stage. In Methods in Mammalian Implantation, pp. 229-245. Ed. J. C. Daniel, Jr. Academic Press, New York.

Kane, M.T. \& Foote, R.H. (1970a) Culture of two- and four-cell rabbit embryos to the blastocyst stage in serum and serum extracts. Biology of Reproduction 2 , $245-250$.

Kane, M.T. \& Foote, R.H. (1970b) Culture of two- and four-cell rabbit embryos to the expanding blastocyst stage in synthetic media. Proceedings of Society for Experimental Biology and Medicine 133, 921-925.

Kane, M.T. \& Foote, R.H. (1971) Factors affecting blastocyst expansion of rabbit zygotes and young embryos in defined media. Biology of Reproduction 4, $41-47$.

Kane, M.T. \& Headon, D.R. (1980) The role of commercial bovine serum albumin preparations in the culture of one-cell rabbit embryos to blastocysts. Journal of Reproduction and Fertility 60, 469-475. 
Kuzan, F.B. \& Wright, R.W. (1982a) Observation on the development of bovine morulae on various cellular and acellular substrata. Journal of Animal Science 54, $811-816$

Kuzan, F.B. \& Wright, R.W. (1982b) Blastocyst expansion, hatching and attachment of porcine embryos cultured with bovine fibroblasts in vitro. Animal Reproduction Science 5, 57-63.

Lear, D., Clarke, A., Gulamhusein, A.P., Huxham, M. \& Beck, F. (1983) Morphological, total nucleic acid and total protein analysis of rat embryos cultured in supplemented and unsupplemented human serum. Journal of Anatomy 137, 279-285.

Lopata, A., Kohlmann, D. \& Johnston, I. (1983) The fine structure of normal and abnormal human embryos developed in culture. In Fertilization of the Human Egg In Vitro, pp. 189-210. Eds H. M. Beier \& H. R. Lindiner. Springer Verlag, Berlin.

McCarthy, S.M., Foote, R.H. \& Maurer, R.R. (1977) Embryo mortality and altered uterine luminal proteins in progesterone-treated rabbits. Fertility and Sterility 28, 101-107.

Mareel, M., Kint, K. \& Meyvisch, C. (1979) Methods of study of the invasion of malignant $\mathrm{C} 3 \mathrm{H}$-mouse fibroblasts into embryonic chick heart in vitro. Virchow's Archives B Cell Pathology 30, 95-111.

Maurer, R.R. \& Beier, H.M. (1976) Uterine proteins and development in vitro of rabbit preimplantation embryos. Journal of Reproduction and Fertility 48, 33-41.

Menezo, Y.J.R., Guerin, J.F. \& Czyba, J.C. (1990) Improvement of human early embryo development in vitro by co-culture on monolayers of Vero cells. Biology of Reproduction 42, 301-306.

Minami, N., Bavister, B.D. \& Iritani, A. (1988) Development of hamster two-cell embryos in the isolated mouse oviduct in organ culture system. Gamete Research 19, 235-240.

New, D.A.T. (1967) Development of explanted rat embryos in circulating medium. Journal of Embryology and Experimental Morphology 17, 513-525.

New, D.A.T. \& Daniel, J.C.R., Jr (1969) Cultivation of rat embryos explanted at 7.5 to 8.5 days of gestation. Nature 223, 515-516.
New, D.A.T., Coppola, P.T. \& Terry, S. (1973) Culture of explanted rat embryos in rotating tubes. Journal of Reproduction and Fertility 35, 135-138.

Nicholas, J.S. (1938) The development of rat embryos in circulating medium. Anatomical Record 70, 199-210.

Potter, S.W. \& Morris, J.E. (1985) Development of mouse embryos in hanging drop culture. Anatomical Record 211, 48-56.

Rexroad, C.E. \& Powell, A.M. (1986) Co-culture of sheep ova and cells from sheep oviduct. Theriogenology 25, 187.

Sanyal, M.S. \& Naftolin, F. (1983) In vitro development of the mammalian embryo. Journal of Experimental Zoology 228, 235-251.

Schumacher, A. \& Fischer, B. (1988) Influence of visible light and room temperature on cell proliferation in preimplantation rabbit embryos. Journal of Reproduction and Fertility 84, 197-204.

Seidel, F. (1960) Die Entwicklungsfähigkeiten isolierter Furchungsstadien aus dem $\mathrm{Ei}$ des Kaninchens Oryctolagus cuniculus. Roux Archiv für Entwicklungsmechanik 152, 43-130.

Sellens, M.H. \& Sherman, M.J. (1980) Effects of culture conditions on the developmental programme of mouse blastocysts. Journal of Embryology and Experimental Zoology 56, 1-22.

Van Blerkom, J., Manes, C. \& Daniel, J.C., Jr (1973) Development of preimplantation rabbit embryos in vivo and in vitro. I. An ultrastructural comparison. Developmental Biology 35, 262-282.

Voelkel, S.A., Amborski, G.F., Hill, H.G. \& Godke, R.A. (1985) Use of a uterine-cell monolayer culture system for micromanipulated bovine embryos. Theriogenology 24, 271-282.

White, K.L., Hehnke, K., Rickards, L.F., Southern, L.L., Thompson, D.L. \& Wood, T.C. (1989) Early embryonic development in vitro by coculture with oviductal epithelial cells in pigs. Biology of Reproduction 41, 425-430.

Whittingham, D.G. (1968) Development of zygotes in cultured mouse oviducts: 2 . The influence of estrous cycle and ovarian hormone upon the development of zygotes. Journal of Experimental Zoology 169, 399-406.

Received 25 March 1991 